\title{
Reported quality of life of people with spinal cord injuries: a longitudinal analysis of the first 6 months post-discharge
}

\author{
Paul Kennedy*,1,2 and Ben Rogers ${ }^{1}$ \\ ${ }^{1}$ Department of Clinical Psychology, National Spinal Injuries Centre, Stoke Mandeville Hospital NHS Trust, \\ Aylesbury, Buckinghamshire, UK, ${ }^{2}$ Isis Education Centre, Warneford Hospital, UK
}

\begin{abstract}
Study design: A repeated measures design was employed with measures taken on two observational periods during the first 6 months post-discharge from hospital.

Objectives: To investigate the perceived importance of specific needs implicated in the development of quality of life (QOL), and the extent to which these aspects have been achieved, in a group of people with spinal cord injury (SCI) living in the community.

Setting: The National Spinal Injuries Centre, Stoke Mandeville Hospital and the general community.

Methods: The study sample comprised 24 spinal cord injured patients discharged from a national rehabilitation centre following a rehabilitation programme. The Quality of Life and Needs Assessment Questionnaire was completed by participants at 1 and 6 months post discharge.

Results: No significant differences were found between the importance that participants attributed to specific needs at months 1 and 6 post discharge. Moreover, no significant differences were found between the extent to which these needs were met at 1 and 6 months post discharge.

Conclusion: The reported quality of life of people who have a spinal cord injury remains stable during the first year following discharge.
\end{abstract}

Spinal Cord (2000) 38, $498-503$

Keywords: spinal cord injury; quality of life; post discharge

\section{Introduction}

The term 'quality of life' (QOL) means a great many different things to different people. Indeed, this difference is reflected both in the numerous definitions applied by researchers, and also in the many instruments used in its measurement. However, despite such conceptual differences, most people will agree that a spinal cord injury constitutes a significant challenge to QOL. ${ }^{1}$ Indeed, since the life expectancy of people who have sustained a spinal cord injury has increased significantly over the last 30 years due to advancements in medical management ${ }^{2}$ QOL issues have become key.

QOL has been examined with reference to a number of specific areas of spinal cord injury. Levels of injury, ${ }^{3}$ time since injury, ${ }^{4}$ age $^{5}$ and pain ${ }^{6}$ have all been implicated in the reduction of QOL. Most modern, empirical research, however, has focused on sources of happiness and satisfaction in persons with a spinal cord injury. Domains in which satisfaction is found to be high include family relationships, living arrangements, social life and passive recreation, whilst

*Correspondence: P Kennedy, Department of Clinical Psychology, National Spinal Injuries Centre, Stoke Mandeville Hospital NHS Trust, Mandeville Road, Aylesbury, Bucks, HP21 8AL, UK domains in which satisfaction is found to be low include finances, sexual life and employment. ${ }^{7-10}$

Quality of life has been measured using a plethora of instruments which in general address similar areas but vary in the breadth and depth of analysis. ${ }^{11}$ Flanagan $^{7}$ developed the Quality of Life and Individual Needs Questionnaire (QOLINQ) as a measure by which quality of life can be quantitatively measured. More than 6500 critical incidents were collected from a sample of nearly 3000 people. Incidents ranged from those that had been especially satisfying, to those that had been harmful, to those that provided the largest change in quality of life. These critical incidents were sorted into specific categories from which, through a process of gradual refinement, 15 quality of life components were formulated. These components were listed under five headings: physical and material well-being; relations with other people; social, community and civic activities; personal development and fulfilment; and recreation. The resulting questionnaire was administered to 2220 non-injured people. Findings indicated that $73 \%$ of the respondents felt that their needs were well met 
in all areas of their lives. Needs reported to be best met were relations with other people and health and personal safety. Needs reported as least well met were social and civic activities, personal development and recreation. The needs that were reported as being most important in relation to quality of life were health and personal safety, having and raising children and understanding oneself. The needs that were reported as being least important in relation to quality of life were participation in local and national government, learning, creative expression and all areas of recreation.

Crewe $^{8}$ administered the QOLINQ to a sample of people who had sustained a spinal cord injury. Results indicated that people with a spinal cord injury reported greater dissatisfaction than had the non-injured sample in Flanagan's study. This was particularly noteworthy with specific reference to: sexual relations with spouse, employment, financial well-being, health and social relationships. These findings have been replicated by a number of studies. ${ }^{6,12-15}$ Indeed, few studies examining older veterans have SCI groups have reported equal or better quality of life than the control group used. ${ }^{16}$ The QOLINQ has also been used with people injured more than 20 years ago with approximately $75 \%$ of participants rating their current quality of life as either good or excellent and similarly so for 10 years previously. However, 20 years previously (nearer to the time of injury) only $56 \%$ of participants rated their quality of life as either good or excellent, suggesting an increase in quality of life over time. ${ }^{4}$

This study aims to examine the reported QOL of patients at two time points during the first 6 months following discharge from a national rehabilitation centre. Flanagan's QOLINQ will be used as a quantitative indicator of QOL since this measure has been used successfully with SCI populations and has been used to examine QOL across time. ${ }^{4,8}$

\section{Methods}

\section{Participants}

The cohort comprised 24 patients (20 males, four females) who were admitted to a national rehabilitation centre for a traumatic spinal cord injury between 1990 and 1994. Inclusion criteria required that participants were between the ages of 16 and 65 when injured, were not private patients and had experienced a traumatic onset of the spinal cord injury.

The mean age of participants was 33.38 years. The primary cause of injury was road traffic accidents, accounting for $45.8 \%$ of injuries. Falls accounted for $25 \%$ of injuries, diving accidents $8.3 \%$, medical problems $12.5 \%$, and sports $8.3 \%$. In general, the demography is similar to data obtained from 66000 American people with spinal cord injuries. ${ }^{17}$
Complete tetraplegic injuries accounted for $16.7 \%$ of all injuries, incomplete tetraplegic injuries accounted for $25 \%$, complete paraplegic injuries accounted for $50 \%$, and incomplete paraplegic injuries accounted for $8.3 \%$ of all injuries.

\begin{abstract}
Materials
Quality of life was measured using the Quality of Life and Individual Needs Assessment Questionnaire (QO$L I N Q)^{7}$ Fifteen dimensions are used to define an individual's quality of life and these are recorded both in terms of the importance that each has to the individual, and in terms of how well each need is met. Participants respond using a five point scale where 1 indicates that the dimension is not important or is not well met, and 5 indicates that the dimension is extremely important or extremely well met. This measure has been used successfully with SCI populations in previous studies. ${ }^{4,8}$
\end{abstract}

\section{Procedure}

Participants from whom consent had been obtained were sent the QOLINQ to complete at 1 and 6 months post-discharge. Those people who required assistance in completing the questionnaire were asked to engage the support of their appropriate personal assistant. Completed forms were returned to the spinal cord rehabilitation centre for analysis.

\section{Results}

Three broad statistical analyses were performed on the data. Primarily, in line with Flanagan's original analysis of the QOLINQ, the percentages of participants who reported each factor as either 'important' or 'highly important' to quality of life were calculated, and the percentages of participants who reported each need as 'well met' or 'very well met' were calculated. These results are illustrated in Table 1. In order to determine whether there were significant differences between the importance that participants attached to each need and the extent to which they felt that this need was met, multiple paired samples $t$-tests were conducted. To further analyze the relationship between the importance and extent to which needs were met, multiple Pearson correlations were performed on the data in order to assess any possible similarities.

\section{Importance of needs}

There are five factors which more than $80 \%$ of participants rated as either important or very important at 1 month post discharge. These were health and personal safety $(95.8 \%)$, relationships with family $(87.5 \%)$, close relationship with spouse/partner $(83.3 \%)$ and close friends $(83.3 \%)$, and work $(87.5 \%)$. At 6 months post discharge the same factors were rated by more than $80 \%$ of people as important or very 
Table 1 Percentage of perceived importance of factor and the extent to which it is achieved

\begin{tabular}{|c|c|c|c|c|}
\hline \multirow[b]{2}{*}{ Factor } & \multicolumn{2}{|c|}{ Month 1} & \multicolumn{2}{|c|}{ Month 6} \\
\hline & Importance & Achieved & Importance & Achieved \\
\hline \multicolumn{5}{|l|}{ Physical and material wellbeing } \\
\hline a. Material comforts & 79.2 & 62.5 & 87.5 & 75.0 \\
\hline b. Health and personal safety & 95.8 & 45.8 & 100.0 & 58.3 \\
\hline \multicolumn{5}{|l|}{ Relationships with other people } \\
\hline c. Relationships with family & 87.5 & 83.3 & 90.7 & 83.3 \\
\hline d. Having and raising children & 75.0 & 41.7 & 58.3 & 37.5 \\
\hline e. Close relationship with spouse/partner & 83.3 & 58.3 & 87.5 & 45.8 \\
\hline f. Close friends & 83.3 & 37.5 & 83.3 & 83.3 \\
\hline \multicolumn{5}{|l|}{ Social community and civic activities } \\
\hline g. Helping and encouraging others & 66.7 & 37.5 & 75.0 & 33.3 \\
\hline h. Participation in activities relating to government & 8.3 & 20.8 & 12.5 & 16.7 \\
\hline \multicolumn{5}{|l|}{ Personal development and fulfilment } \\
\hline i. Learning-attending school & 37.5 & 16.7 & 41.7 & 16.7 \\
\hline j. Understanding yourself & 75.0 & 50.0 & 79.2 & 54.2 \\
\hline k. Work & 87.5 & 45.8 & 83.3 & 41.7 \\
\hline 1. Expressing yourself & 33.3 & 25.0 & 41.7 & 29.2 \\
\hline \multicolumn{5}{|l|}{ Recreation } \\
\hline m. Socialising & 58.3 & 54.2 & 58.3 & 66.7 \\
\hline $\mathrm{n}$. Reading, listening to music & 45.8 & 58.3 & 50.0 & 58.3 \\
\hline o. Participation in active recreation & 37.5 & 50.0 & 41.7 & 33.3 \\
\hline
\end{tabular}

important: health and personal safety $(100 \%)$, relationships with family (91.7\%), close relationship with spouse/partner $(87.5 \%)$ and close friends $(83.3 \%)$ and work $(83.3 \%)$. However, material comforts was rated by $87.5 \%$ of participants at month 6 as either important or very important which was greater than its score of $79.2 \%$ for month 1 .

Five factors were rated by between $50 \%$ and $80 \%$ of participants as either important or very important for month 1 . These were material comforts $(79.2 \%)$, having and raising children (75\%), helping and encouraging others $(66.7 \%)$, understanding yourself $(75 \%)$, socialising $(58.3 \%)$. At 6 months post discharge, the same factors (with the exception of material comforts) were rated by between $50 \%$ and $80 \%$ of participants as either important or very important. These were having and raising children $(58.3 \%)$, helping and encouraging others $(75 \%)$, understanding yourself $(79.2 \%)$ socialising $(58.3 \%)$. Also, reading/listening to music $(50 \%)$ was included.

Six factors were rated by less than $50 \%$ of participants as either important or very important for month 1 . These were participation in activities relating to government $(8.3 \%)$, learning, (37.5\%) expressing yourself $(33.3 \%)$, reading/listening to music $(45.8 \%)$ and participation in active recreation $(37.5 \%)$. As at month 1, at 6 months post discharge the same six factors were rated by less than $50 \%$ of participants as either important or very important for month 1 . These were participation in activities relating to government $(12.5 \%)$, learning $(41.7 \%)$, expressing yourself $(41.7 \%)$ and participation in active recreation $(41.7 \%)$.
In order to examine whether changes had occurred in the mean importance people had attached to individual needs at months 1 and 6, multiple paired samples $t$-tests were conducted between the importance attached to a need at month 1 and the importance attached to the same need at month 6 . Due to the large number of tests conducted, the significance level was adjusted to 0.01 . No significant differences were identified for any of the needs. Indeed, none of the $t$-test approached significance at any level.

Extent to which needs are met

In contrast to the five factors rated as either important or very important at 1 month post discharge, only relationships with family $(83.3 \%)$ was rated by more than $80 \%$ of participants as either well met or very well met at this time point. At 6 months post discharge only relationships with family (83.3\%) was still regarded as either well met or very well met by more than $80 \%$ of participants. Moreover, close friends $(83.3 \%)$ was also rated as either well met or very well met by more than $80 \%$ of participants.

At 1 month post discharge, six factors were rated as either well met or very well met by between $50 \%$ and $80 \%$ of participants: material comforts $(62.5 \%)$, close relationship with spouse/partner $(58.3 \%)$, understanding yourself $(50 \%)$, socialising $(54.2 \%)$, reading/listening to music $(58.3 \%)$ and participation in active recreation $(50 \%)$. At 6 months, five factors were similarly rated: material comforts $(75 \%)$, close relationship with spouse/ partner $(45.8 \%)$, understanding yourself $(54.2 \%)$, 
socialising $(66.7 \%)$, reading/listening to music $(58.3 \%)$ and participating in active recreation $(33.3 \%)$.

Four factors were rated as either well met or very well met by less than $50 \%$ of participants at 1 month post discharge. These were helping and encouraging others $(37.5 \%, 33.3 \%)$, participation in activities relating to government $(20.8 \%, 16.7 \%)$, learning $(16.7 \%, 16.7 \%)$, work $(45.8 \%, 41.7 \%)$ and expressing yourself $(25 \%, 29.2 \%)$. These four factors were also rated as either well met or very well met by less than $50 \%$ of participants at 6 months post discharge: helping and encouraging others $(33.3 \%)$, participation in activities relating to government $(16.7 \%)$, learning $(16.7 \%)$, work $(41.7 \%)$ and expressing yourself $(29.2 \%)$.

In order to examine whether changes had occurred in the mean importance people had attached to the extent to which individual needs were met at months 1 and 6, multiple paired samples $t$-tests were conducted between the extent to which a need was met at month 1 and the extent to which the same need was met at month 6. Due to the large number of tests conducted, the significance level was adjusted to 0.01 . No significant differences were identified for any of the needs.

\section{Differences in importance of need and extent to which met}

To determine whether there were significant differences between the importance that participants attached to each need and the extent to which they felt that this need was met, a series of paired samples $t$-tests were conducted. Due to the large number of tests conducted, the significance level was adjusted to 0.01 .

At 1 month post discharge significant differences were identified for health and personal safety $(t=6.811$, $P<0.001)$, having and raising children $(t=2.796$,

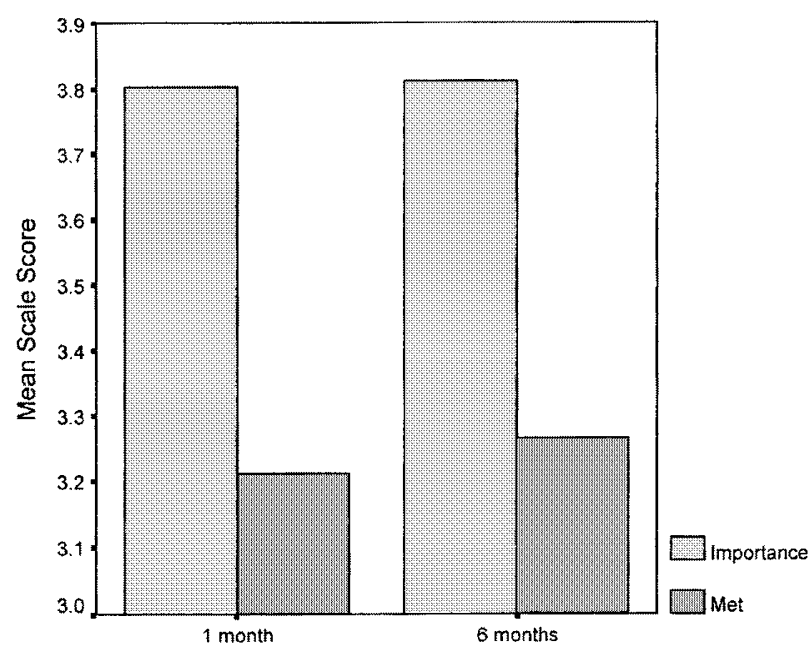

Figure 1 Mean importance and extent to which met ratings for needs at month 1 and month 6
$P<0.05)$, close relationship with spouse/partner $(t=3.206, \quad P<0.005), \quad$ learning/attending $\quad$ school $(t=3.423, P<0.005)$, work in a job or at home that is interesting, rewarding, worthwhile $(t=4.164, P<0.001)$, participating in active recreation $(t=2.991, P<0.01)$. For all cases where significant differences were identified, the importance of the need was greater than the extent to which it was met.

At 6 months post discharge significant differences were identified for health and personal safety $(t=5.318$, $P<0.001)$ relationships with family $(t=2.846$, $P<0.01)$, close relationship with spouse/partner $(t=4.303, \quad P<0.001)$, helping and encouraging others $(t=3.243, \quad P<0.005), \quad$ learning-attending school $(t=5.000, P<0.001)$, understanding yourself $(t=2.849$, $P<0.01)$, work in a job or at home that is interesting, rewarding, worthwhile $(t=4.486, P<0.001)$. As with month 1 , for all cases where significant differences were identified, the importance of the need was greater than the extent to which it was met.

\section{Similarities in importance of need and extent to which met}

The $t$-tests illustrated above present a number of significant differences between the importance which participants to a specific need, and the extent to which they report that need as being met. However, to further assess the relationship between these two variables, multiple correlations have been implemented. Due to the large number of tests conducted, the significance level was adjusted to 0.01 .

At 1 month post discharge significant correlations were identified for relationships with family $(r(24)=0.660, P<0.001)$, close friends $(r(24)=0.559$, $P=0.005), \quad$ learning-attending school $\quad(r(24)=0.674$, $P<0.001)$, expressing yourself in a creative manner $(r(24)=0.636, \quad P=0.001), \quad$ socialising-meeting other people $(r(24)=0.711, P<0.001)$ and reading, listening to music $(r(24)=0.701, P<0.001)$.

At 6 months post discharge significant correlations were identified for close friends $(r(24)=0.749$, $P<0.001)$, helping and encouraging others $(r(24)=0.521, \quad P=0.009), \quad$ learning-attending school $(r(24)=0.674, \quad P<0.001), \quad$ socialising $\quad(r(24)=0.642$, $P=0.001)$ and reading/listening to music $(r(24)=0.629, P=0.001)$.

It is significant to note from Table 1 and from the analyses illustrated above, that the mean importance attributed to each of the needs were generally greater than the extent to which each need was met. Figure 1 illustrates the mean 'importance' and 'extent to which met' scores for the whole cohort. Inspection of Figure 1 reveals that the importance attributed to the needs for both month $1 \quad($ mean $=3.8)$ and month 6 $($ mean $=3.8)$ was greater than the extent to which needs were met during month 1 (mean 3.2) and month 6 (mean $=3.3$ ). Moreover, it can be seen from the graph that these scores remain stable across both time points. 


\section{Discussion}

A number of critical findings can be drawn from the data illustrated. Primarily, it is the relative stability of the importance of individual needs and the extent to which those needs are met at both 1 and 6 months post discharge. Thus, it would seem apparent that QOL as measured by the QOLINQ remains stable during the first 6 months post discharge. Secondly, the importance that people attached to specific needs is consistently greater than the extent to which those needs were met, suggesting that people's needs are not being met effectively post discharge. Finally, the findings highlight critical needs for people who have sustained a spinal injury living in the community during the first 6 months post discharge, and the extent to which these needs are met.

The needs that were consistently reported to be the most important were health and personal safety, relationships with family, close relationship with spouse/partner, close friends and work. These findings confirm those identified by Flanagan et al, ${ }^{7}$ who also found that health and personal safety, close relationship with spouse/partner and work were the most important factors for people. However, Flanagan et al, ${ }^{7}$ also identified that material comforts and having and raising children were of great importance. This is not borne out from the results of this study. Moreover, there is some discrepancy between the most important needs identified within this study and those identified by Whiteneck et al, ${ }^{4}$ who ascertained that expressing yourself, reading and listening to music to be the most important needs. Indeed, expressing yourself was considered to be one of the least important needs within this study. The least important needs consistently reported were participation in activities relating to government, learning, expressing yourself and participation in active recreation. Likewise, Flanagan et al, ${ }^{7}$ illustrated that participation in activities relating to local government, expressing yourself and participation in active recreation were all needs which people felt were least important. Whiteneck et al, ${ }^{4}$ support the notion that participation in civic activities was not considered to be important.

The only need that was consistently reported to be well met was relationships with family. Indeed, none of the remaining needs were reported to be well met. This is not consistent with the findings of Flanagan et $a l^{7}$ in which health and personal safety, close relationships with spouse/partner, having and raising children were consistently reported to be well met. The findings of Whiteneck et al, ${ }^{4}$ indicate that reading and listening to music, observing sporting events and entertainment were well met for SCI patients. Needs that were reported to be worst met were helping others, participation in activities relating to local government, learning, and expressing yourself. Needs that were least well met are similar to those found by Whiteneck et $a l,{ }^{4}$ namely social community and civic activities, personal development and having and raising chil- dren. However, this study, like Flanagan's non-injured population, identified that recreation was not found to be well met, although at 6 months socialising had increased somewhat.

Comparisons made between the importance that people attached to specific needs and the extent to which those needs were met identify that at 1 month post discharge there are significant discrepancies between importance and achievement for health and personal safety, having and raising children, close relationship with spouse/partner, learning/attending school, work and participating in active recreation. Since similar data is not available for either Flanagan's or Whiteneck's studies, comparison with other groups is not possible. However, this information highlights critical areas of need for people who have a spinal cord injury and are living in the community. Like month 1 , at 6 months postdischarge significant discrepancies were identified for health and personal safety, close relationship with spouse/partner and learning-attending school. However, a number of specific changes had occurred. Primarily, the mean rated importance for having and raising children was reduced from $75 \%$ to $58 \%$ and as such, the discrepancy between this and the extent to which it was met was reduced. Discrepancies for relationships with family also emerged which did not reflect the extent to which this need was fulfilled (this remained stable) but the mean importance which was increased. Helping and encouraging others and understanding yourself were also identified at month 6 since both were rated as more important at month 6 than at month 1.

A number of significant clinical implications can be drawn from these findings. Foremost, the stability of both the importance of needs and the extent to which these needs are met, suggests that an assessment of QOL made at 1 month post discharge could be used as an indicator of QOL at 6 months post discharge. However, the subtle variance noted between months 1 and 6 indicated in the differences between the importance of each need and extent to which each need is met, suggests that there are specific changes which occur in thinking and may therefore affect QOL. Thus, whilst QOL remains stable over the first 6 months post discharge, the clinician should be aware of the possibility of change in QOL during this time. A further clinical implication is the identification of specific needs of people with spinal cord injury that are not met in the community. Namely, helping others, participation in activities relating to local government, learning, and expressing yourself. Moreover, those needs for which the importance is greater than the extent to which the needs are met indicate key areas that may attribute to diminished QOL. Clinicians should therefore be aware of differences in expectations/attributed importance of a need and the extent to which it is met.

There are a number of problems associated with this study which need to be addressed in interpreting 
results gained. Principally, the QOLINQ was developed in 1978. It could therefore be argued that it represents QOL issues that were most salient during the 1970's and as such, is an unrepresentative scale for use 20 years on. Secondly, the cohort comprises 20 males and only four females. Whilst this is representative of the spinal cord injured population, it makes comparison with Flanagan's original cohort problematic and the comparisons made in this study should therefore be treated with some caution.

The problems highlighted above, coupled with the clinical and theoretical implications provide a number of areas in which future research should be conducted. Firstly, this study represents a first step in the longitudinal analysis of QOL following spinal cord injury. Future studies are now needed which address this issue over longer periods of time including the acute phase of care and rehabilitation. In doing so, causal mechanisms of high QOL might be better established and interventions developed which are able to help individuals improve existing levels of QOL. Secondly, it has been suggested that the small number of females in this study makes comparison with Flanagan's study problematic. An alternative analysis of these results might exclude the females from the sample and compare the results with the subset of 30 year-old male participants used in Flanagan's study. Since the mean age of the 20 men in this study is 32 , Flanagan's subset would prove a useful non-injured comparison group. Finally, since Flanagan's QOLINQ was developed in 1978 it might be argued that new measures should be incorporated within new designs investigating QOL following SCI. New QOL measures such as Lundqvist et al' $\mathrm{s}^{18}$ brief quality of life (QL) questionnaire might therefore be more appropriate scales for use in such designs.

In conclusion, the data suggests that QOL remains stable during the first 6 months post discharge. However, the subtle variance noted between months 1 and 6 with specific reference to the differences between the importance of each need and the extent to which each need is met, suggests that there are changes which occur in people's internal attributes and external circumstances. These changes may subsequently have an attributing role in QOL and should therefore be acknowledged within clinical practice.

\section{Acknowledgements}

The authors gratefully appreciate all of those patients who kindly co-operated in this study. Thanks also to Emma Short who was involved in data analysis and Linda Hall for her typing.

\section{References}

1 Dijkers M. Quality of life after spinal cord injury: A meta analysis of the effects of disablement components. Spinal Cord 1997; 35: 829-840.

2 Geisler WO, Jousse AT, Wynne-Jones M, Breithaupt D. Survival in traumatic spinal cord injury. Paraplegia 1983; 21: 364-373.

3 Clayton KS, Chubon RA. Factors associated with the quality of life of spinal cord injured persons. Arch Phys Med Rehabil 1994; 75: $633-638$

4 Whiteneck GG et al. Mortality, morbidity and psychosocial outcomes of persons with spinal cord injury more than 20 years ago. Spinal Cord 1992; 30: 617-630.

5 Stensman R. Adjustment to traumatic spinal cord injury: A longitudinal study of self-reported quality of life. Spinal Cord 1994; 32: $416-422$.

6 Lundqvist $\mathrm{C}$ et al. Spinal cord injuries: Clinical, functional and emotional status. Spine 1991; 16: 17-83.

7 Flanagan JC. A research approach to improving our quality of life. Am Psychol 1978; 13: $138-147$.

8 Crewe N. Quality of life: The ultimate goal in rehabilitation. Minn Med 1980; 163: 586-589.

9 Krause JS. Life satisfaction after spinal cord injury: A descriptive study. Rehabil Psychol 1992; 37: 61-70.

10 Tate DG, Dijkers M, Johnson-Greene L. Outcome measures in quality of life. Top Stroke Rehabil 1996; 2: 1-17.

11 Wright S. Health status assessment. In: Baum A et al (eds) Cambridge handbook of psychology, health and medicine. Cambridge: Cambridge University Press, 1995.

12 Decker SD, Schultz R. Correlates of life satisfaction and depression in middle-aged and elderly spinal cord injured persons. Am J Occup Ther 1985; 39: 740-745.

13 Boschen KA. Life satisfaction, housing satisfaction and locus of control: A comparison between spinal cord injured and nondisabled individuals. Can J Rehabil 1990; 4: 75-88.

14 Crisp R. Long term adjustment of 60 persons with spinal cord injury. Aust Psychol 1992; 27: $43-47$.

15 Fuhrer MJ et al. Relationship of life satisfaction to impairment, disability and handicap among persons with spinal cord injuries. Arch Phys Med Rehabil 1992; 73: 552 - 557.

16 Eisenberg MG, Salts CC. Quality of life among spinal cord injured persons: Long term rehabilitation outcomes. Spinal Cord 1991; 29: $514-520$.

17 Stover SL, Delisa JA, Whiteneck GG. Spinal Cord Injury: Clinical Outcomes from the Model Systems. Aspen Publishers, 1995.

18 Lundqvist C et al. Spinal cord injuries: a shortened measure of function and mood. Spinal Cord 1997; 35: 17-21. 